\title{
OPERACIONES DE CONSERVACIÓN Y MANTENIMIENTO EN LA PUERTA DE LOS APÓSTOLES DE LA CATEDRAL DE VALENCIA, ESPAÑA
}

\author{
PRESERVATION AND MAINTENANCE WORKS IN THE PUERTA DE LOS APÓSTOLES OF THE \\ CATHEDRAL OF VALENCIA, SPAIN)
}

Julían Esteban Chapapría, Dr. Arquitecto (Jefe del Servicio de Arquitectura de la Generalitat Valenciana); col: Rafael Soler Verdú, Dr. Arquitecto

Fecha de recepción: 16-VIII-96

\section{RESUMEN}

Se examinan -de forma explicita- las operaciones necesarias que han de realizarse con respecto a la conservación y mantenimiento de la Puerta de los Apóstoles de la Catedral de Valencia, posteriores a la restauración que, en 1992, fue llevada a cabo por parte de diversas instituciones y empresas colaboradoras.

\section{SUMMARY}

The works that must be carried out and the necessary steps to be taken regarding preservation and maintenance of the Puerta de los Apóstoles in the cathedral of Valencia are thoroughly examined. They are the follow up of those carried out in 1992 by several firms and institutions working together.
"En noviembre de 1992, y tras dieciocho meses de trabajos, finalizaban una serie de tareas encaminadas a la conservacióny puesta en valor de la Puerta delos Apóstoles de la Catedral de Valencia" (Fig. 1). Así comenzaba la reseña que en esta misma revista se publicó sobre la intervención realizada y que de manera sintética abordaba algunas de las operaciones practicadas ${ }^{1}$.

Al cabo de unos meses, la zona de las arquivoltas del cuerpo bajo comenzó a evidenciar la aparición de una serie de patologías: pérdida del patinado, erosión de aristas, etc. (Figs. 2, 3 y 4), que han preocupado no solamente a los técnicos queintervenimos en la restauración, sino también a la opinión pública, aunque desgraciadamente en los términos de escándalo en los que en España, y desde el

(1) Informes de la Construcción, Vol. $45 n^{\circ} 427$, septiembre/octubre 1993. J. ESTEBAN CHAPAPRÍA: "Algunas notas sobre la restauración de la Puerta de los Apóstoles de la Catedral de Valencia (España)".

(c) Consejo Superior de Investigaciones Científicas Licencia Creative Commons 3.0 España (by-nc)

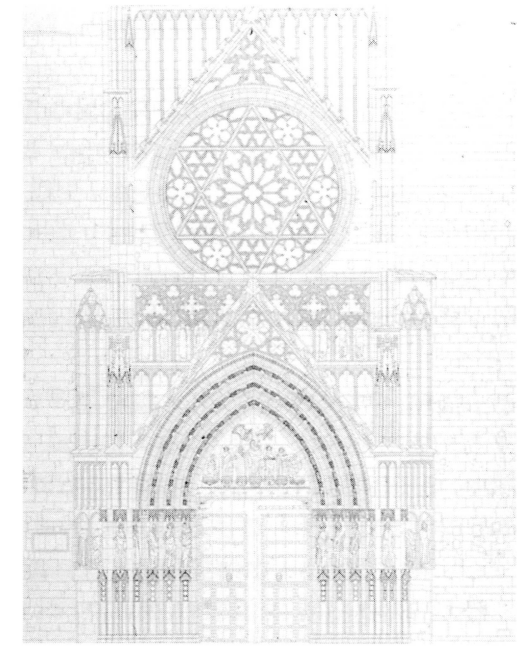

Fig. 1.- Alzado de la Puerta de los Apóstoles de la Catedral de Valencia, realizado por el Servicio de Arquitectura de la Conselleria de Cultura de la Generalitat Valenciana. 


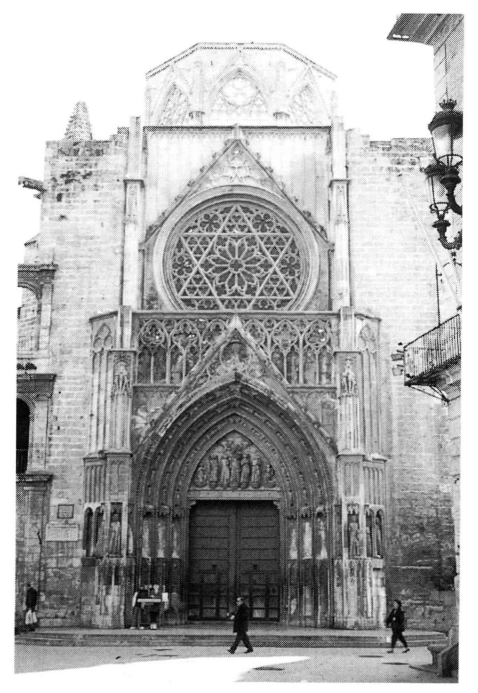

Fig. 2.- Estado previo a la restauración iniciada en marzo de 1991.

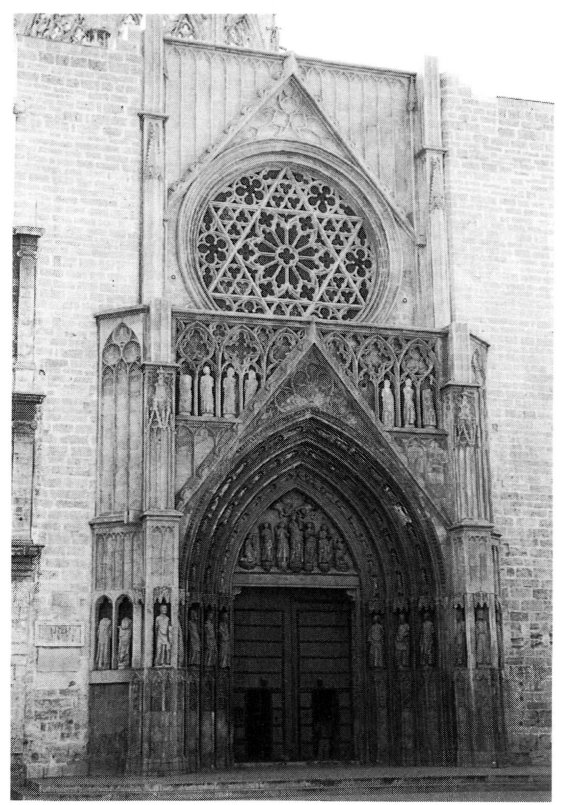

Fig. 4.- Estado actual de la portada (julio 1996).

siglo pasado, son tratados los temas de patrimonio. Este artículo pretende abordar, con el rigor que la cuestión requiere, la actuación específica que en 1992 se llevó a cabo en esa parte de la portada, las causas y sintomas de la degradación reaparecida, así como las opciones de conservación y mantenimiento que se están realizando.

1.- En primer lugar, debe recordarse que la Puerta de los Apóstoles se construye en la primera mitad del s. XIV como un elemento de renovación, sustituyendo a otra ya existente sobre el testero del crucero del evangelio. Ello condicionó, lógicamente, su morfología constructiva, ya que mientras el cuerpo alto se desarrolla en el espesor de la fábrica preexistente, el cuerpo bajo, con un programa formal mucho más complejo, necesitó una mayor superficie de desarrollo que se obtuvo proporcionando mayor espesor a esta parte de la construcción, creando con (c) Consejo Superior de Investigaciones Científicas Licencia Creative Commons 3.0 España (by-nc)

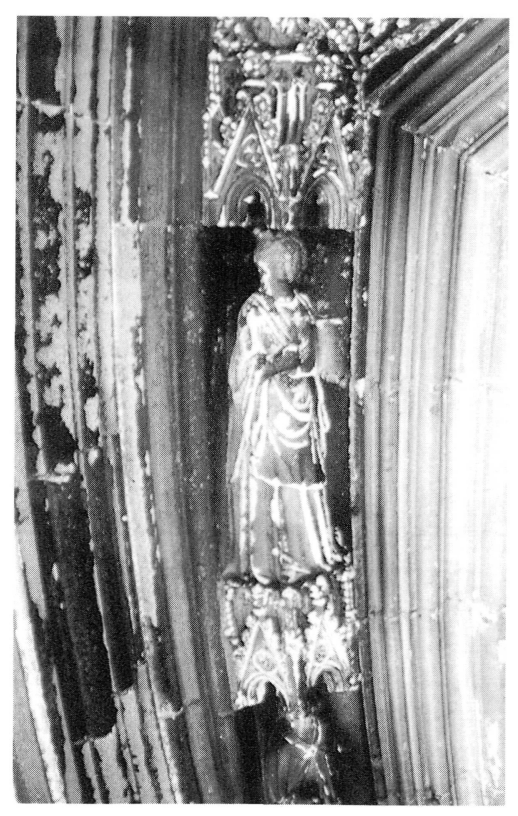

Fig. 3.- Detalle de la degradación de la arquivolta, previo a la restauración.

ello una terraza que cubría la superficie que el cuerpo bajo se adelantaba respecto al alto.

Desde su origen, la evacuación de aguas de esta terraza se realizaba interiormente a través del relleno que componía el espacio existente entre las arquivoltas y la terraza, hasta buscar las gárgolas que se encontraban tres metros más abajo en los laterales de la portada. La obturación de esta canalización, y en general de todo el sistema de evacuación, hacía que el agua almacenada en la terraza se filtrara a través del relleno de cascotes y mampuestos con argamasa de cal que componía el macizado, llegando por gravedad hasta las arquivoltas y produciendo costras, arenización, pérdidas de superficie y de pátina.

Una segunda cuestión de gran importancia, quizás la fundamental, es la piedra con la que está ejecutada la parte ornamental de la Portada. A diferencia de la caliza travertínica utilizada en los laterales de piedra franca, se trata de una caliza blanca de grano medio, calcarenita sumamente porosa, fácil de labrar, pero también débil frente a unas condiciones ambientales o físicas agresivas. Los acabados con estucos y policromías le proporcionaron, mientras los tuvo, una protección nada desdeñable, pero que requerían un continuo mantenimiento, como así demuestran los archivos ${ }^{2}$. Los efectos de la degradación no tardaron en hacerse notar: aquellos elementos decorativos de la Portada más expuestos, como la tracería calada con la macolla que remataba el cuerpo superior o la que existía también sobre el cuerpo bajo sobre el falso triforio, fueron demolidas a finales del s. XVI debido a quesu descomposición provocabala continua caída de cascotes sobre el atrio, con el consiguiente peligro para los que por allí circulaban ${ }^{3}$. 
En mayor o menor grado, las otras dos portadas de la Catedral: la románica, conocida como de la Almoina, y la barroca, conocida como de los Hierros, presentan problemas de la misma indole, reflejo de una problemática común: la del deterioro de los materiales pétreos expuestos a la intemperie y sometidos a la acción destructiva de los agentes climáticos en un ambiente contaminado. La románica, que se conservaba en buen estado hasta hace escaso tiempo, manifiesta, de forma incipiente, los primeros sintomas de degradación superficial de la piedra, siendo en la puerta barroca donde este proceso ha avanzado a tal velocidad que, en muchas partes, puede considerarse irreversible (Fig. 5). Su estado y la caída de cascotes condujo, en febrero pasado, al montaje de un andamio y realización de estudios encaminados a la conservación. Los análisis realizados por el Instituto de Conservación y Restauración de Bienes Culturales (ICRBC), que se van a hacer extensivos a la de los Apóstoles, ha proporcionado información de sumo interés sobre características y patologías de la piedra calcarenita empleada, de similares características a la empleada en la puerta de los Apóstoles, por ejemplo el estado de descohesión intergranular de la roca y su traducción en un extraordinario aumento de la porosidad abierta y una notable pérdida de sus propiedades mecánicas, así como el espectacular crecimiento de

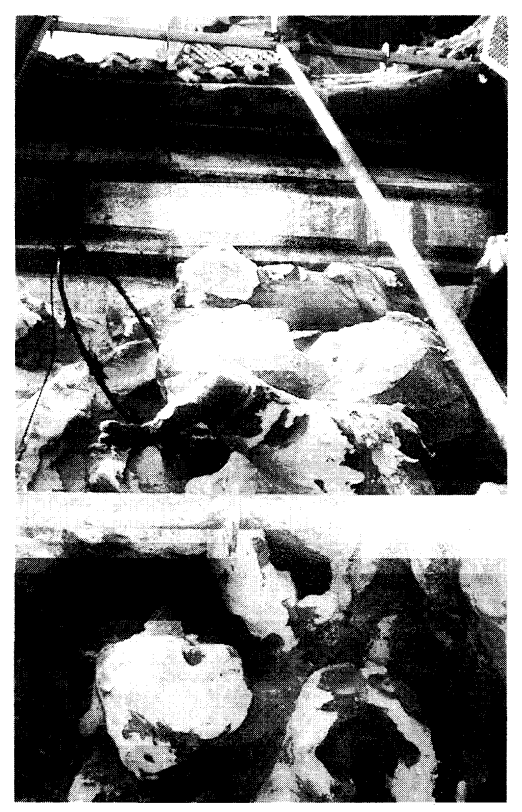

Fig. 5.- Puerta barroca de la Catedral, conocida como de los Hierros. Estado de degradación de uno de los grupos escultóricos principales. cristales de singenita, que parecen ser los responsables directos del avanzado estado de deterioro de la caliza. De ninguna manera, los daños existentes parecen deberse a la limpieza y tratamiento que, en 1982, con motivo de la visita de Juan Pablo Ila la ciudad, se practicó a esta portada, como "especialistas", con interés en hacer estudios y promocionar sus métodos, han pretendido señalar.

2.- En la restauración de la Puerta de los Apóstoles, realizada a partir de 1967 por los arquitectos Joan Segura de Lago y Alejandro Ferrant (Fig. 6), se mantuvo el sistema de evacuación de la terraza del cuerpo bajo, canalizándolo a través de dos bajantes de fibrocemento, alojadas en el interior de los muros y conectadas con dos pequeñas gárgolas situadas lateralmente en la intersección de los contrafuertes de la portada y sus fachadas laterales; se realizó una solera sobre el relleno original, pavimentándose con baldosa cerámica su superficie y creando unos imbornales protegidos. La defecación y cadáveres de palomas inutilizó pronto la terraza, por falta de mantenimiento. Además, la zona de arquivoltas fue objeto de un tratamiento de limpieza, del que desgraciadamente no se guarda memoria escrita u oral, ya que la mayoría de sus protagonistas han fallecido.

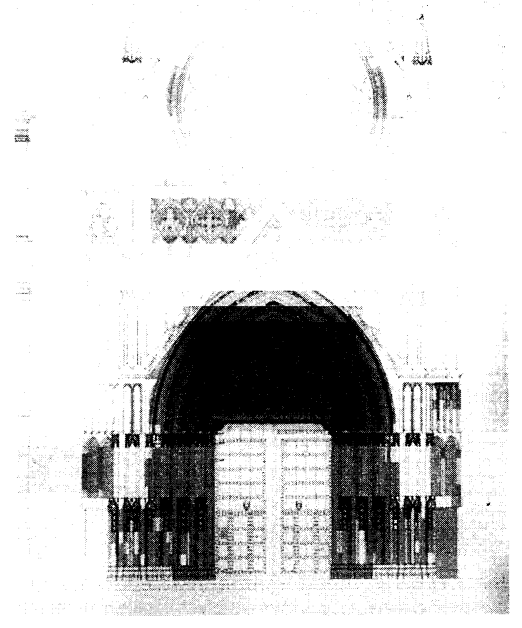

Fig. 6.- Restauración realizada en 1967 por los arquitectos Segura de Lago y Ferrant. En oscuro, la sillería no sustituida.

(2) Asientos de pago, concernientes a las obras de reparación y pintura de la Puerta de los Apóstoles de la Catedral de Valencia, concertadas con el mestre pintor Miguel Alcanyis. A.C.V.: Llibre de la obra de la Seu de Valencia, año 1431 fol. $28_{r}^{\circ}$, sig. 1.479. Asiento de pago a Joan Joaquin, comerciante, por los colores y aglutinantes empleados en la pintura de las imágenes de la Puerta de los Apóstoles en la Catedral de Valencia. 1432, diciembre, 31 .

A.C. V.: Protocolo del notario de Jaume Monfort del año 1432 sig. 3.531. fol. 1 ${ }_{r}^{\circ}$. Asientos depago a Francesc Baldomar, maestro cantero, Nicolau Borrell, Valenti i mestre Jordi, pintor, por los trabajos de cantería y pintura efectuados en la renovación del parteluz de la Puerta de los Apóstoles de la Catedral de Valencia. 1470, enero, 18/1470, abril, 9.A.C.V.: Llibre dela fábrica de 1469-1470 fol. $18 \mathrm{v}^{\circ}$, sig. 1482

(3) Albarán y asiento de pago efectuado a Vicent Esteve, maestro cantero, por los trabajos efectuados en la demolición de los apitradores de la Puerta de los Apóstoles de la Catedra de Valencia. 1597, marzo 27. A.C. V.: Libre de la fábrica de 1596, fol. $26 \mathrm{~V}^{\circ}$, Sig. 
Cuando en 1990 se dio comienzo a los estudios y prediagnóstico sobre la puerta quellevaron a cabo técnicos de la empresa Coresal, la pérdida de pátina y descomposición volumétrica en las dos arquivoltas exteriores eran de nuevo bien patentes. El análisis petrográfico practicado fue completado durante la ejecución de las obras con el análisis de otras cinco muestras estratigráficas, realizado por el Dr. José $\mathrm{M}^{\mathrm{a}}$ Cabrera, y que proporcionó un conocimiento a fondo de la cuestión (Fig. 7). Además, con el fin de disponer de una información adecuada de la relación existente entre la humedad relativa exterior y la conductividad de la molduración y figuras de las arquivoltas, fueron tomados dichos parámetros tres veces al día, durante cuatro días de los meses de mayo, junio y septiembre de 1992, de seis figuras y seis puntos del conjunto de arquivoltas, elaborando los consiguientes gráficos y obteniendo referencia de los tratamientos que se estaban ejecutando.

Las operaciones realizadas entre marzo de 1991 y noviembre de 1992, en aquello que se refiere al problema del cuerpo bajo y de la degradación de las arquivoltas, pueden resumirse en dos: las actuaciones de carácter constructivo y los tratamientos superficiales aplicados a las figuras y molduración de las arquivoltas. De entre las primeras, las actuaciones de carácter constructivo, además de la creación de drenajes y barreras perimetrales que impidieran la ascensión de la humedad de capilaridad, y de operaciones de corrección y reintegración que facilitaran una buena evacuación del agua de lluvia, se actuó sobre la terraza del cuerpo bajo. En ella, los trabajos comenzaron por el levantamiento dela terraza realizada en la restauración de 1972, dejando al descubierto la parte inferior del anillo exterior del rosetón que había quedado oculto al realizar la terraza. Se pudo comprobar que se trataba de un relleno inerte y compacto, realizado en origen, a base de un hormigón de cal con mampuestos, bolos, ripios y

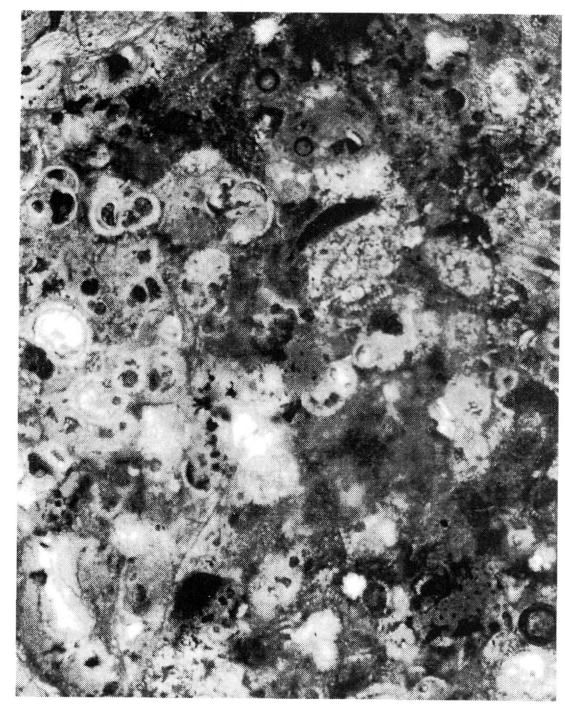

Fig. 7.- Análisis petrográfico practicado sobre una muestra de material de las arquivoltas, realizado por la empresa C. P. A., S. L.

(c) Consejo Superior de Investigaciones Científicas fragmentos desechados de piedra labrada, que actuaba como una auténtica esponja, reteniendo importantes cantidades de agua quelentamente seevaporaba a través de la sillería de las arquivoltas, proceso en el que las sales solubles arrastradas en vapor de agua y su posterior cristalización provocaban la destrucción de la cohesión de la piedra. Si a ello se añade la absorción continua de vapor de agua, proveniente de la alta humedad relativa ambiental que tiene la ciudad, cuya media anual en Valencia ciudad es del $70 \%$, podemos deducir los riesgos de degradación a los que está expuesta la piedra de esta parte de la portada.

Ante esta situación, y dadas sus pequeñas dimensiones, se optó por renovar totalmente la cubierta, único modo de resolver los graves defectos apuntados. Se planteó como necesario rebajar el plano general de evacuación respecto al punto inferior del rosetón, para tener, al menos, treinta centímetros de borde lateral del vaso estanco y, complementariamente, aumentar la pendiente. Durante esta operación en la azotea inaccesible se pudo observar, por primera vez, las características heterogéneas del relleno, tanto de los materiales como de su distribución y consistencia.

Esta operación pretendía la eliminación del trasdós húmedo que pudiera transmitir agua hacialos paramentos del cuerpo inferior de la portada, ya que es ésta una de las principales causas del deterioro. La cámara así creada, se ventila para conseguir que el relleno inferior pueda eliminar el exceso de humedad y crear unas condiciones de autorregulación higrotérmica y de equilibrio.

La nueva azotea proyectada se despiezó mediante una limatesa situada en el eje transversal, con sendos faldones que vierten las aguas a cazoletas que se sitúan en ambos laterales, ligeramente separadas de los muros para permitir una adecuada solución de encuentro en todo su perímetro. Constructivamente, el vaciado inferior fue resuelto volteando arquillos de ladrillo macizo apeados sobre el rebanco del relleno trasdós de los muros. Superiormente, un tablero cerámico y una capa de mortero de cemento formaron el soporte base. La membrana impermeabilizante no adherida fue reforzada perimetralmente con una lámina autoprotegida en todo el perímetro, elevándose más de $25 \mathrm{~cm}$ respecto al plano de evacuación. Una capa de aislante de poliestireno extrusionado, tenía como misión proteger de los cambios térmicos a la membrana con el fin de frenar el envejecimiento, siendo la protección pesada una capa de grava rodada. Mediante los mechinales dejados en el paramento y las chimeneas de ventilación dispuestas sobre el tablero se consigue un grado de ventilación elevado de la cámara creada con el vaciado realizado. El conjunto fue sometido a pruebas de estanquidad, mostrando su correcta ejecución, funcionando hasta el momento sin problemas (Figs. 8 y 9 ). 


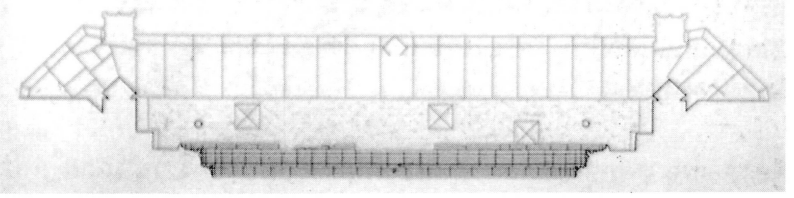

Fig. 8.- Planta de la terraza superior del cuerpo bajo objeto de la intervención constructiva, con indicación de chimeneas de ventilación.

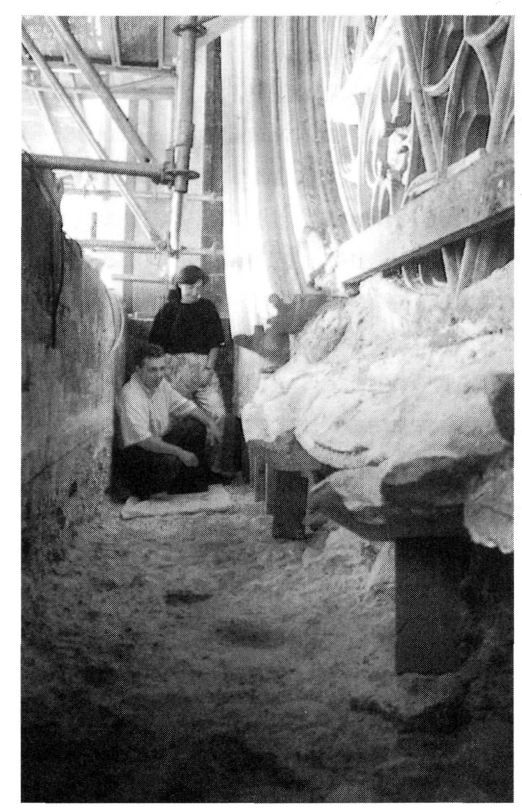

Fig. 9. - Proceso de vaciado del relleno de la terraza superior del cuerpo bajo.

Respecto a la segunda de las operaciones practicadas en esta parte de la Puerta, los tratamientos superficiales, debe mencionarse que de la observación del cuerpo bajo cabe clasificar en dos grandes apartados el comportamiento del material pétreo de la misma:

a) una zona en el que la piedra ha conservado los revestimientos tradicionales, aunque de muy diversa técnica y época, pero que impedían o disminuían a valores insignificantes la entrada de vapor de agua,

b) otra zona en el que la piedra, carente de cualquier revestimiento, presentaba su superficie desnuda, desprotegida y en estado de descomposición. En ella, después de los pertinentes trabajos de consolidación, fue dotada de una protección, incidiendo en que no impidiera la natural transpiración de la piedra. Para no recurrir a los revestimientos de estucos o impregnaciones de aceite, que podían llevar a confusión con revestimientos históricos, se decidió aplicar resinas acrílicas en una operación neutra y reversible.

Nada mejor puede explicar el proceso que la documentación obranteen la Memoria final elaborada, que, de manera sucinta, expone los datos referentes al proceso de conocimiento y conservación realizado, consistente,

(c) Consejo Superior de Investigaciones Científicas

Licencia Creative Commons 3.0 España (by-nc) básicamente, en tratamientos de preconsolidación, limpieza, extracción de sales, consolidación y entonado ${ }^{4}$.

3.- Medio año después de finalizar la intervención se manifestaban, como ya se ha indicado, los primeros síntomas de que el proceso de degradación de la piedra no se había podido detener con los procedimientos aplicados (Figs. 10 y 11). Se produjeron despellejaduras "alifaces”, a modo de lagunas, en una parte de la zona afectada por esa patología antes de la intervención restauratoria. Debe señalarse que, mientras que en las primeras manifestaciones la película desprendida no contenía apenas cantidad sensible de material pétreo, presentando la superficie desnuda de la piedra un buen estado de consolidación, en las últimas observaciones practicadas, además de una mayor extensión de la degradación en las dos arquivoltas exteriores, ésta ha comenzado a afectar a la piedra en un lento proceso de arenización superficial.

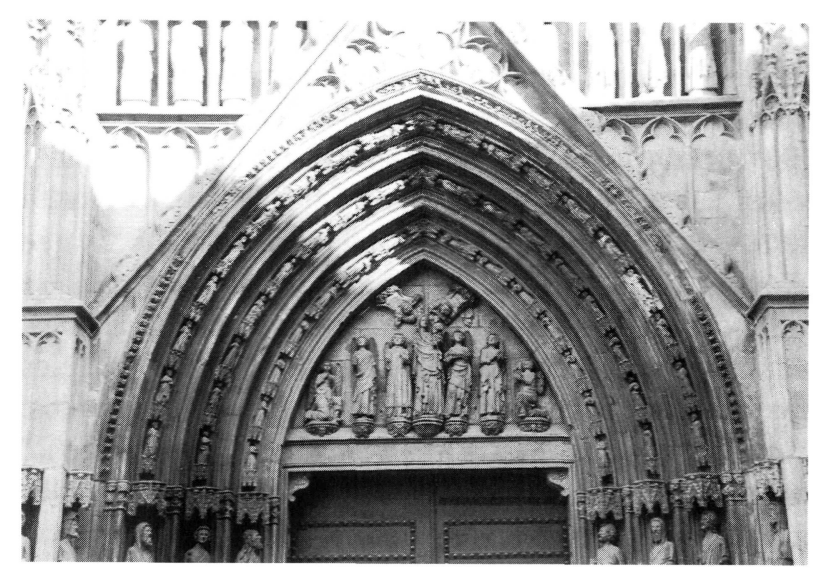

Fig. 10.- Conjunto de arquivoltas y tímpano, donde pueden apreciarse las áreas superficialmente degradadas.

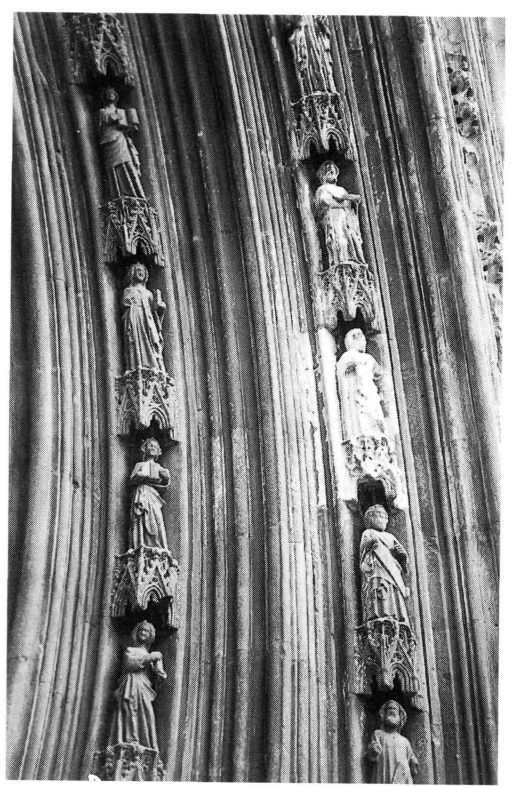

Fig. 11.- Detalle de la arquivolta exterior derecha, con pérdida de pátina y degradación superficial.

(4) Ver anexo con las fichas correspondientes a las arquivoltas. 
El origen de la degradación subyace, a nuestro entender, en la calidad y características de la piedra utilizada en la construcción de la portada y la causa se debe a una mayor o menor exposición a los agentes atmosféricos (lluvia otoñal, viento y humedad relativa) de determinados elementos arquitectónicos finamente trabajados. De esta manera, las dos arquivoltas exteriores están sometidas a una mayor agresión que la interior, protegida totalmente por el abocinamiento y que conserva todavía un estuco de protección. Si se examinan fotografias anteriores a la restauración de 1967, este fenómeno es bien patente para las zonas más expuestas o con mayor superficie labrada (Fig. 12).

Es preciso realizar, pues, dos puntualizaciones: la primera, quela degradación no se debe a un rechazo o empeoramiento por el tratamiento aplicado, que en el resto de sectores se ha mostrado eficaz, o a un mal funcionamiento de la terraza, que evacúa correctamente y sin pérdidas, como ha podido observarse durante este tiempo. La segunda es que frente a una opción de intervención, seguida en tantos países y en tantas restauraciones, entre ellas la realizada en 1967 en esta misma Puerta, de sustituir cuidadosamente la piedra dañada o degradada por otra piedra nueva, de mano de talleres permanentemente instalados a pie de obra y que se ocupan de la continua reparación de las Catedrales, se prefirióla aplicación de tratamientos menos contundentes, aún con la convicción de que hay procesos irreversibles que sólo, y en el mejor de los casos, pueden ser ralentizados. El camino iniciado en esta intervención supone, de hecho, un cambio en la manera de actuar en relación con todas las actuaciones anteriores, ya que se trata de una intervención basada en operaciones de carácter conservativo, centradas

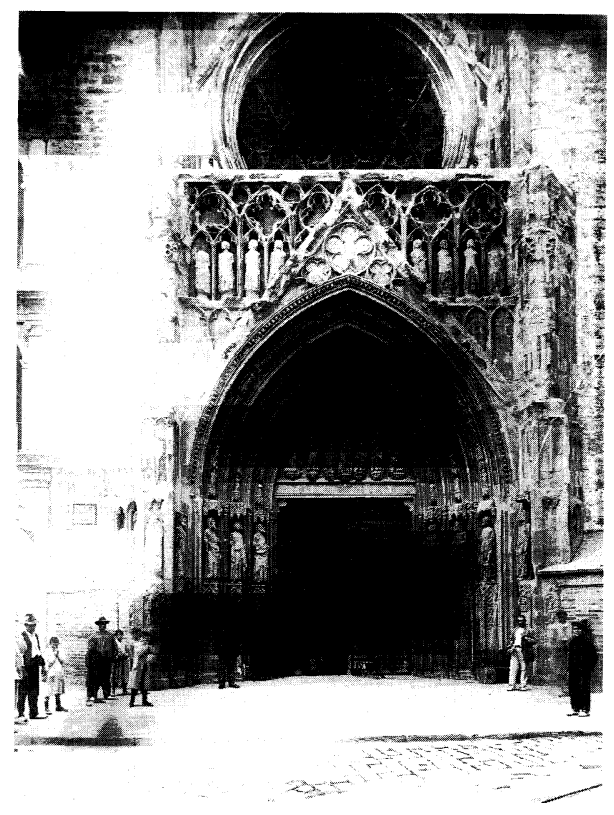

Fig. 12.- Puerta de los Apóstoles en una toma fotográfica de 1911 (foto Arxiu Mas).

(c) Consejo Superior de Investigaciones Científicas en preservar la integridad material, reversibles, y complementadas con trabajos de puesta en valor de su arquitectura.

Respecto a los daños aparecidos con posterioridad a la restauración, tras la eliminación de las despellejaduras con pincel y brocha, limpieza y nueva extracción de sales, se aplicó, con el asesoramiento del Dr. José M $M^{a}$ Cabrera, un tratamiento de protección, consistente en una disolución al $10 \%$ en White Spirit de resina acrílica y cera $n^{\circ} 90$ al $0,7 \%$ respecto a la resina sólida, y un entonado general a base de pigmentos naturales disueltos en acetona. Operaciones de consolidación y protección que habrá que repetir periódicamente hasta conseguir frenar la degradación, completando las actuaciones realizadas en el momento de la restauración.

Se entra, de esta manera, en una dinámica de mantenimiento sobre la que merece hacer alguna consideración de orden teórico. La puesta en valor de la arquitectura histórica ha venido, en los últimos decenios, consolidando el término conservación como el conjunto global de acciones y medidas a realizar sobre aquellos edificios que componen el patrimonio colectivo y el medio ambiente construido de una determinada sociedad. Este término, por extensión, ha pretendido sustituir al de restauración, acuñado en el siglo XIX, y que representaba un muy particular modelo de intervención, caracterizado por suponer, la mayoría de las veces, operaciones de fuerte incidencia constructiva, estructural o compositiva.

El término conservación comprende, entre muchas otras, las operaciones de carácter preventivo. Son éstas las que aquí interesan como alternativa a aquellas otras, en ocasiones no menos necesarias, pero siempre más traumáticas, costosas y de mayor riesgo para el monumento. Las actuaciones de mantenimiento son, en esencia, operaciones que se ocupan de mantener el bien después de haberlo construido o de haberlo restaurado con cuidados y técnicas de simple reparación o mínima renovación. Precisamente la historia de la Puerta de los Apóstoles demuestra la idoneidad de este tipo de actuaciones, ya que fue objeto, desde su construcción hasta el s. XVI, de un atento y continuado cuidado que la debió mantener en un envidiable estado de conservación; sus problemas comenzaron alli donde no fueron aplicadas o cuando fue abandonada su ejecución. Frente a esta opción nos encontramos con su más reciente historia: restauraciones en 1950,1970 y 1990 que superan con creces el de operaciones de mantenimiento.

Sin duda existen diversas maneras de llevar a cabo el atento cuidado y vigilancia de los monumentos, algunas puestas ya en marcha por la Dirección General de Patrimonio Artístico de la Generalitat Valenciana, como la practicada por los arquitectos conservadores. Pero el ejercicio de la conservación y el mantenimiento a la que aquí pretende 
hacerse referencia, frente a otras posibles, es aquélla que debe realizarse tras la restauración. Marconi ${ }^{5}$, ha señalado claramente cómo el mantenimiento es "la otra cara de la arquitectura" y comienza allí donde termina la restauración. Efectivamente, puede afirmarse que, tras intervenciones de consolidación o restauración, con lo que suponen de acercamiento al conocimiento histórico y material del objeto arquitectónico, se está en una disposición óptima para la planificación y ejecución de operaciones de mantenimiento adecuadamente diseñadas al caso. Los fines son varios:

- mantener en correcto estado de conservación tanto las partes intervenidas como las que se consideraron sanas,

- comprobar la compatibilidad eidoneidad delas operaciones realizadas, queen algunos casos requerirán de posterior tratamiento o aplicación,

- realizar tratamientos en un período dilatado de tiempo, superior al de una obra normal, que eviten la aplicación de técnicas más traumáticas o extrañas a la sustancia intervenida, permitiendo la erradicación de problemas dificilmente abordables de otra manera,

- controlar las acciones agresivas medioambientales o humanas, de sobreuso, abandono o de expoliación que pudieran ocurrir,
- profundizar en el conocimiento del monumento intervenido, continuando las investigaciones iniciadas.

Con todo, la cultura de la conservación requiere de la conjunción de diversos factores: la concienciación de los usuarios o poseedores de ese patrimonio, la existencia de canales y medios administrativos al alcance de los responsables de la tutela patrimonial y, por último, la consolidación de un arte y una técnica del mantenimiento ya de por sí de dificil adquisición en relación con la arquitectura histórica.

Sobre estos principios básicos es sobre los que se propuso, a la Dirección General de Patrimonio Artístico de la Generalitat Valenciana, la elaboración de un Plan de Conservación y Mantenimiento de la Puerta de los Apóstoles de la Catedral de Valencia, tras la restauración realizada. Tanto laimportancia dela pieza, la delicadeza de las operaciones ejecutadas y la convicción de que algunos de los problemas existentes deben ser objeto de resolución a medio plazo, están conduciendo a la formulación del Plan, documento que se encuentra en fase de redacción y que se espera disponer en pocos meses. Su contenido hará referencia, como especifica el pliego de condiciones técnicas con el que ha sido encargado, a la descripción arquitectónica de la portada, memoria de las intervenciones realizadas, objetivos del Plan, operaciones a realizar, calendario de actividades e informes. 


\title{
PUERTA DE LOS APÓSTOLES. CATEDRAL DE VALENCIA
}

\author{
CÓDIGO DE IDENTIFICACIÓN: 6.A ARQUIVOLTAS
}

FECHA: 04 de 1991 / 11 de 1992

\section{IDENTIFICACIÓN}

1.1 Nombre de la escena, escultura o motivo : Arquivoltas

1.2 Situación dentro del conjunto de la obra: Cuerpo bajo

\section{HISTORIATÉCNICA}

\section{OBSERVACIONES GENERALES PREVIAS AL TRATAMIENTO}

\subsection{Breve descripción del objeto}

Se encuentran situadas en el cuerpo bajo, conformando el vano de ingreso. Son una serie de arapuntados, que disminuyen de tamaño cuanto más al interior se encuentran, en los que alternan los que no disponen de decoración con los que disponen de motivos escultóricos. Estos últimos son 3 y están formados por figuras coronadas por doseletes, que muestran tres caras vistas, que sirven de peana para los superiores. Estos doseletes son similares a los doseles de los apóstoles, aunque de menor tamaño, y se encuentran profusamente decorados.

Las figuras se distribuyen de la siguiente forma: La arquivolta exterior cuenta con un total de 18 tallas distribuidas, como en las restantes, mitad en cada lateral. Las figuras representan profetas, que sostienen entre sus manos bien libros o bien pergaminos (mayoritariamente). La arquivolta intermedia está formada por sibilas, en número de 16 , sujetando libros. La arquivolta interior dispone de 14 ángeles ceroferarios.

\subsection{Marcas e inscripciones}

\subsubsection{Marcas de cantero: NO}

\subsubsection{Inscripciones: NO}

\subsection{Estado material}

\subsubsection{Estado de conservación}

Presentan notables diferencias según en la arquivolta en la que se encuentren, aunque es común a todas ellas las pérdidas de volumen, así como parte de las esculturas, principalmente brazos, atributos y cabezas

La arquivolta más interior (ángeles) se encuentra en bastante buen estado, excepto lo anteriormente apuntado. El resto presenta graves problemas de costras en la capa de protección y desconchados, que se separan en finas láminas, dejando a la vista superficies arenizadas. Esto se acusa más en la arquivolta exterior, al perder importantes partes la moldura de protección de la misma. Asimismo, en algunos puntos (juntas), se pueden observar florescencias.

\subsubsection{Aspecto general (situación en esquema)}

3.3.2.1 Fisuras: SÍ

3.3.2.2 Grietas: SI

3.3.2.3 Roturas: SI

3.3.2.4 Pérdidas: Zonas de molduración. Brazos, atributos y cabezas en las figuras. También partes de los doseletes

\subsubsection{Soporte}

3.3.3.1 Tipo: Piedra sin ningún tipo de protección en las zonas repuestas. Restos de pátina original en arquivoltas y tallas.

3.3.3.2 Estado conservación: MALO

3.3.3.3 Observaciones: El estado en general es malo, a excepción de la $1^{\mathrm{a}}$ arquivolta que se encuentra en buen estado.

\section{EXAMEN DE LABORATORIO}

\subsection{Objetivo}

a) Identificación de los distintos tipos de piedra que pudiesen existir. Para ello se han obtenido 6 láminas delgadas de otros tantos trozos de roca, empleándose para el estudio un Microscopio Universal de Investigación de la firma ZEISS, equipado con un sistema automático de fotografia. La identificación taxonómica se amplia con los parámetros más interesantes desde el punto de vista del estudio: porosidad, fracción arenosa no carbonatada, minerales básicos y accesorios y fósiles.

b) Identificación de los distintos tipos de policromía que pudiesen existir, realizándose un estudio microscópico y microquímico de varias micromuestras.

Dado el tipo de muestras encontrado, se ha realizado un estudio simultáneo, tanto de la piedra como de las diversas capas de protección o policromías existentes, por lo que el número de muestras y los códigos asignados son el mismo en todos los apartados. En cada uno de ellos expondremos los resultados más relevantes obtenidos de cada una de las micromuestras (lámina delgada).

\subsection{Análisis petrográfico}

\subsubsection{Número de muestras: 1}

4.2.2 Código asignado: I-AAI6 / PI-1 


\subsubsection{Caracterización petrográfica:}

Caliza fosilifera arenosa.

Los fósiles están constituidos, fundamentalmente, por foraminiferos (muy recristalizados), nummulites, globigerinas, radiolas y equinodermos, representando, en conjunto, el $92 \%$ de la roca.

La fracción arenosa (5\%) es fina (tamaño medio $0,1 \mathrm{~mm}$ ) y su mineralogía está constituida por cuarzo, feldespato y clastos de chert con filosilicatos. Es notable la abundancia de glauconita $(3 \%)$. También se observan, en menor cantidad, óxidos de hierro y opacos como minerales accesorios. La porosidad es escasa.

\subsection{Material del soporte}

4.3.I Número de muestras: 4

4.3.2 Código asignado: I-AAD7 / PO-2, I-AAI5 / PO-3, I-ASD4 / PO-4, I-APD7 / PO-5

\subsubsection{Identificación:}

Diversas figuras de las arquivoltas: Ángel 7 de la derecha, Ángel 5 de la izquierda, Sibila 4 de la derecha, Profeta 7 de la derecha.

\subsubsection{Composición:}

Piedra impregnada sobre medio oleaginoso, en el que se identifican, fundamentalmente, sólo lípidos.

\subsection{Policromía: NO}

\subsection{Capa de protección}

\subsubsection{Número de muestras:}

\subsection{2 (ódigo asignado (localización en esquema)}

Solamente aparece en la arquivolta más interior, correspondiente a la de los ángeles.

\subsubsection{Identificación}

Capa color blanco de estuco, imitando mármol

Capa superficial color siena

\subsubsection{Composición}

Capa color blanco de estuco, imitando mármol: Estuco de cal. Capa color siena: Pintura de yeso y pigmentos ocre y rojo minio.

\section{TRATAMIENTO PROPUESTO}

\subsection{Limpieza superficial}

\subsubsection{Método}

Eliminación de polvo superficial, materia orgánica procedente de aves y suciedad general, mediante aspiración por aire, cepillado suave con brocha y levantamientos superficiales con espátula blanda.

\subsubsection{Materiales empleados}

Aspirador, brocha, espátula blanda

\subsubsection{Dosificación}

\subsection{Fijación previa: SÍ}

\subsubsection{Método}

\subsubsection{Materiales}

A elegir entre los siguientes productos:

*TEGOVAKON V / CONSOLIDANTE H = Silicatos de etilo, parcialmente hidrolizados en disolvente orgánico (White Spirit), con aproximadamente el $40 \%$ en $\mathrm{SiO}_{2}$

*PARALOID B72 / SYNOCRYL 9122X = Esteres polimetacrílicos parcialmente hidrolizados en disolvente orgánico (White Spirit), con aproximadamente $40 \%$ de contenido en resina sólida.

\subsubsection{Dosificación}

*SYNOCR YL 9122X en disolución al 2,5\% en NITRO o XILENO/ White Spirit.

\section{*PARALOID B72}

\subsection{Tratamiento fungicida}

\subsubsection{Método:}

Extracción suave con cepillo y espátula blanda de los líquenes, seguido de tratamiento con disolución de formol (formalina) hasta su completa erradicación.

\subsubsection{Materiales empleados}

Espátula blanda, cepillo, formalina.

\subsubsection{Dosificación}

Formalina en disolución al 20-30\%.

\subsection{Sales}

\subsubsection{Método}

Se aplicarán emplastos de pasta absorbente, extendida mediante brocha suave y dejando que actúe por impregnación/ absorción, durante un plazo entre 24 y 48 horas.

\subsubsection{Materiales empleados}

PASTA $=$ celulosa + arcillas (atapulgita, caolín $)+$ tensoactivo aniónico (cloruro de bario) + agua.

5.4.3 Dosificación

$\begin{array}{ll}\text { Celulosa } & 1 \text { parte } \\ \text { Arcilla } & 2 \text { partes, }\end{array}$

Cloruro de bario $\quad 1 \%$

Agua hasta homogeneizar la pasta 


\subsection{Limpieza}

\subsubsection{Método}

Aplicación con nebulizador de la disolución, frotado con cepillo suave, aclarado con agua. Lavado final con White Spirit (proporciona la eliminación del el velo blanquecino que aparece tras la limpieza).

Este método se emplea para la limpieza general de todas las superficies.

\subsubsection{Materiales empleados}

Disolución formada por alcohol + amoníaco + agua desionizada.

\subsubsection{Dosificación}

$\begin{array}{ll}\text { Alcohol } & 60 \% \\ \text { Amoníaco } & 3 \% \\ \text { Agua desionizada } & 32 \%\end{array}$

5.6 Retacado de juntas y sellado de grietas

\subsubsection{Método}

Se efectuará mediante mortero de resina epoxídica (cicloalifática) previa impregnación de la zona a sellar con acetona, que facilita un mejor agarre. El producto base seria MOWILITH

\subsubsection{Materiales empleados}

MORTERO $=$ cemento blanco $+\mathrm{cal}+$ marmolina + sílice.

\subsubsection{Dosificación}

Cemento blanco 1 parte

$\begin{array}{ll}\text { Marmolina } & 8 \text { partes } \\ \text { Cal } & 2 \text { partes } \\ \text { Sílice } & 2 \text { partes }\end{array}$

\subsection{Reintegración}

\subsubsection{Método}

Se realizarán mediante emparrillado de varilla roscada de fibra de vidrio de $8 \mathrm{~mm}$ y refuerzos de $6 \mathrm{~mm}$. El mortero será de base tradicional, con adición de modificante acrílico para mortero COTELATEX o PRIMAL AC-33 y con adición de pigmentos naturales.

\subsubsection{Materiales empleados:}

Mortero $=$ cemento blanco + cal + marmolina + sílice fina + sílice gruesa + pigmentos naturales + COTELATEX.

PRIMAL AC-33= Resina acrílica de la misma naturaleza que el PARALOID, preparado en forma de emulsión en agua.

COTELATEX= Preparado acrílico de naturaleza similar.

\subsubsection{Dosificación}

Cemento blanco 1

Sílice gruesa $\quad 2$

Cal 3

Sílice fina $\quad 1$

Marmolina 9

Pigmentos naturales: hasta dar color.

COTELATEX: Hasta homogeneizar la pasta, según rutina de trabajo.

5.8 Nivelación: Ídem. procedimiento 5.7

5.9 Consolidación: Ídem. procedimiento preconsolidación.

\subsection{Patinado final}

\subsubsection{Método}

3 capas: de fondo, entonación y acabado. Tierras disueltas en acetona, aplicadas con brocha y muñequillas.

En zonas cuyo fondo es muy oscuro, previa la aplicación de estas 3 capas se aplicará una mano inicial compuesta por acetona y blanco litopón.

\subsubsection{Materiales empleados}

Acetona; pigmentos: siena natural, negro vid, blanco litopón, siena calcinada; brochas.

\subsubsection{Dosificación}

$1^{a}$ Capa (Sólo en las arquivoltas, basas y doseles).

1 I de acetona

$6 \mathrm{~g}$ de blanco litopón.

\section{Capa de fondo}

2 I de acetona.

$11 \mathrm{~g}$ pigmento siena natural.

$2,5 \mathrm{~g}$ pigmento negro de vid.

$6 \mathrm{~g}$ pigmento blanco litopón.

\section{Capa entonación}

2 I acetona.

$11 \mathrm{~g}$ siena natural.

$10 \mathrm{~g}$ negro de vid.

$24 \mathrm{~g}$ blanco litopón.

13 g siena calcinada.

\section{Capa acabado}

2 I acetona.

20 g negro vid.

5.11 Eliminación de morteros degradados 


\subsubsection{Método}

Se efectuarán por procedimientos mecánicos, nunca químicos, utilizando escoplos de tamaños adecuados. Se pondrá especial atención en respetar las aristas de los sillares.

\subsubsection{Materiales empleados}

Escoplos.

\subsubsection{Dosificación}

\subsection{Hidrofugación}

\subsubsection{Método}

Aplicación del producto en cortina mediante nebulizador, hasta saturación.

\subsubsection{Materiales empleados}

Zonas con erosión: TEGOSIVINHL-100.

Zonas sin erosión: DRYFILM 104. TEXA-FOB SILICON. CONSOLIDANTE OH.

TEGOSIVIN HL-100 / CONSOLIDANTE OH: Son silicatos de etilo + silicona, parcialmente polimerizados.

DRY-FILM / TEXSA FOB SILICON: Son etil-siliconas en White Spirit.

ACRIL-SILICONA (Paraloid B72 o Synocryl + Dry Film o Texsa-fob Silicon).

Se propone: Preconsolidar con silicato de etilo o Hidrofugar con silicato + silicona.

\subsubsection{Dosificación}

En todos los casos, mediante dilución conveniente en White Spirit.

\subsection{Fijación y sentado estucos}

\subsubsection{Método}

Se llevará a cabo mediante preconsolidación con PARALOID B-72 disuelto en White Spirit y/o Xileno/ White Spirit, mediante aplicación con pincel de pelo suave.

\subsubsection{Materiales empleados}

Pincel pelo suave.

PARALOID B-72, Nitro/White Spirit, Xileno/White Spirit.

\subsubsection{Dosificación}

PARALOID B-72 disuelto al 2,5\% en Nitro/White Spirit. Xileno/White Spirit.

\subsection{Limpieza químico - mecánica de estucos, dorados} y policromías

\subsubsection{Método}

\subsubsection{Materiales empleados}

ESTUCOS: Alcohol; amoníaco; aguarrás, esencia de trementina o similar.

POLICROMIAS: Dimetil formamida; alcohol/acetato de amilo; nitro.

DORADOS: Alcohol; decapante.

\subsection{Consolidación de estucos y protección final}

\subsubsection{Método}

Se llevará a cabo según lo descrito en los puntos "FIJACIÓN PREVIA"e "HIDROFUGACIÓN".

\section{TRATAMIENTOS APLICADOS}

\subsection{Fijación previa}

\subsubsection{Causas modificación}

No se aprecia efectividad con la disolución propuesta.

\subsubsection{Nuevo método empleado}

El método es el mismo aumentando el número de aplicaciones, así como la concentración de la disolución en las mismas. Se realizan las primeras aplicaciones generales con nebulizador, insistiendo posteriormente en zonas puntuales con pincel.

\subsubsection{Materiales empleados}

Nebulizador y pincel.

TEGOVAKON V varias concentraciones en la disolución.

\subsubsection{Dosificación}

$1^{\circ}$.- 2 capas superficiales al 2,5\%.

3 capas a pincel al $2,5 \%$.

$2^{\circ}$ - 1 aplicación con nebulizador al $5 \%$.

2 aplicaciones a pincel al $5 \%$.

$3^{\circ}$.-aplicaciones con pulverizador al $15 \%$.

$4^{\circ}$.-1 aplicación con pulverizador al $20 \%$.

$5^{\circ} .-2$ aplicaciones con pincel al $30 \%$.

\subsection{Tratamiento fungicida. Método previsto}

\subsection{Extracción de sales. Método previsto}

\subsection{Limpieza. Método previsto}

\subsection{Retacado de juntas y sellado de grietas}

\subsubsection{Causas de la modificación}

Para el retacado de juntas se empleará mortero (descrito en 5.6.2) + COTELATTEX (50\% agua).

Para el sellado se empleará mortero (descrito en 5.6.2) + pigmentos naturales + MOWILITH-50.

\subsubsection{Nuevo método empleado}

En algunos casos de sellado de grietas, y de acuerdo a la 
importancia de las mismas, o estado de la zona donde se encuentran, se añaden unos cosidos con varilla de fibra de vidrio, rellenándose estas perforaciones con MOWILITH + gel de sílice, o marmolina, más o menos fluido, según casos

\subsubsection{Materiales empleados}

Mortero descrito en 5.6.2:

+ COTELATEX

+ MOWILITH-50 + pigmento

Retacado.

Sellado.

Varilla de fibra de vidrio de $3 \mathrm{~mm}+$ MOWILITH-50 + gel de silice

MOWILITH-50 + marmolina

Sellado.

\subsubsection{Dosificación}

Mortero: descrito en punto 5.6.2

Retacado: mortero + COTELATEX (en disolución al en agua). $50 \%$

Sellado: mortero + MOWILITH-50 en disolución al + pigmentos. $15 \%$

\subsection{Reintegración}

\subsubsection{Causas modificación}

Aparición de grano demasiado grueso para la realización del retalle.

\subsubsection{Nuevo método empleado}

Se ha empleado el mismo método, variando las proporciones de la mezcla.

\subsubsection{Materiales empleados}

COTELATEX $=$ Preparado acrílico, en forma de emulsión en agua

MORTERO $=$ Cemento blanco $+\mathrm{cal}+$ marmolina + sílice fina + sílice gruesa + pigmentos naturales + gel de sílice + COTELATEX.

\section{6.+ Dosificación}

\section{Cemento blanco $\quad 0,5$}

Marmolina 8

Cal 3

Sílice fina 2

Gel de sílice 1

Sílice gruesa 1

COTELATEX: $50 \%$ agua de amasado.

Pigmentos naturales: hasta dar color.

\subsection{Nivelación}

Ídem. punto anterior 6.6

\subsection{Consolidación}

\subsubsection{Causas modificación}

Tras la aplicación del consolidante previsto inicialmente, se observa una falta de efectividad en diversos puntos. 6.8.2. Nuevo método empleado

El consolidante aplicado inicialmente fue TEGOVAKON $\mathrm{V}$ (sin diluir), aplicado mediante pulverizador e insistiendo con pincel en aquellos puntos más degradados. Dada la falta de efectividad del mismo en ciertas zonas, comprobada tras un mes, aproximadamente, de la realización del tratamiento, se decidió la aplicación de SYNOCRYL disuelto en White Spirirt, mediante pulverización e insistiendo a pincel en aquellos puntos donde se considera necesario.

\subsubsection{Materiales empleados}

TEGOVAKONV,SYNOCRYL, WhiteSpirit, pulverizador, pincel.

\subsection{Dosificación}

TEGOVAKON V sin diluir.

SYNOCRYL en disolución con White Spirit al 2,5\%.

\subsection{Patinado final}

\subsubsection{Causas modificación}

Se procedió inicialmente como estaba previsto, pero en aquellos puntos donde el consolidante no ha sido efectivo, se han producido pérdidas de las capas de entonación, repitiéndose el proceso.

\subsection{Eliminación de morteros degradados. Método previsto}

\subsection{Hidrofugación:}

\subsubsection{Causas de la modificación}

Se realizó inicialmente la aplicación de TEGOSIVIN HL100 disuelto en White Spirit. Transcurrido un mes desde la finalización completa de los tratamientos, se detectó en ciertas zonas de las arquivoltas la no efectividad del consolidante, procediéndose a un nuevo tratamiento, expuesto en los apartados correspondientes, finalizando conla aplicación de SYNOCR YL disuelto en White Spirirt.

\subsubsection{Nuevo método empleado}

1ªplicación: TEGOSIVIN HL-100 disuelto en White Spirit, aplicado mediante nebulizador.

$2^{\mathrm{a}}$ aplicación: SYNOCRYL 9122X disuelto en White Spirit, aplicado mediante nebulizador.

\subsubsection{Materiales empleados}

TEGOSIVIN HL-100, SYNOCRYL 9122X, White Spirit, nebulizador.

\subsubsection{Dosificación}

$\mathbf{1}^{\mathrm{a}}$ aplicación: TEGOSIVIN HL-100 diluido al $10 \%$ en White Spirit. 
$2^{\text {a }}$ aplicación: SYNOCRYL 9122X diluido al 2,5\% en White Spirit.

\subsection{Fijación y sentado estucos. Método previsto}

6.13 Limpieza químico mecánica de estucos, dorados y policromías. Método previsto

6.14 Consolidación de estucos y protección final. Método previsto

Publicaciones del Instituto Eduardo Torroja - CSIC

Número monográfico de MATERIALES DE CONSTRUCCIÓN

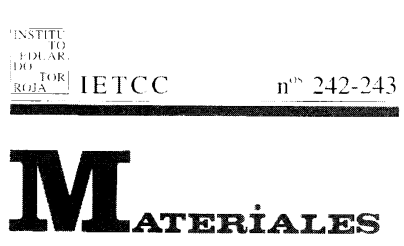

EL VIDRIO EN LA CONSTRUCCION

DE

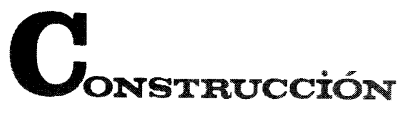

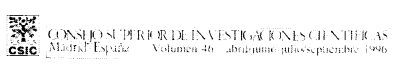

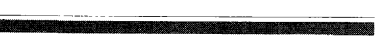

EL VIDRIO EN LA CONSTRUCCIÓN

(n $\left.{ }^{\circ} 242-243\right)$
- Procesos de alteración de las vidrieras medievales. Estudio y tratamientos de protección. (Alteration processes of medieval stained glass windows. Study and protection treatments).

J. M $M^{a}$ Fernández Navarro.

- El efecto de la corrosión en vidrieras coloreadas.

(The effect of corrosion of stained glass windows).

J. Leissner.

- Formulación de vidrios absorbentes del calor. (Formulation of heat absorbing glasses).

P. Álvarez-Casariego y P. Mazón.

- Dimensionamiento de placas de acristalamientopara edificios mediante un modelo probabilístico de rotura. (A probabilistic model for failure design of glass plates in building).

A. Fernández Canteli. I. Viña y A. Bernardo Sánchez

- Caracterización de fibras en forma de lana de roca para aislamiento obtenidas a partir de basaltos canarios. (Characterization of fibers as rockwool for insulation obtained from canary islands basalts).

J. M. Cáceres, J. E. García Hernández y J. Mª Rincón.

- El GRC: Material compuesto de matriz inorgánica reforzado con fibras de vidrio AR.

(GRC: Composite material from an inorganic matrix reinforced with ar glass fibres).

P. I. Comino.

- Los materiales vitrocerámicos en la construcción

(Glass-ceramic as building materials).

J. Min Rincón y M. Romero.

- Gres porcelánico: Aplicaciones arquitectónicas, procesado y propiedades físico-mecánicas. (Porcelainized stoneware: Architectural, processing and physico-mechanical properties). T. Manfredini, M. Romagnoli y J. Ma Rincón.

Venta de ejemplares: distribución de Publicaciones

Instituto de Ciencias de la Construcción Eduardo Torroja

c/Serrano Galvache, s/n - 28033 Madrid

Tfno.. (91)302.04.40-Fax: (91) 302.07.00 